Nachdruck:

\title{
Die Bedeutung der
} sowjetischen Neuropathologie und Psychiatrie für die Neugestaltung der Fachrichtung während des Aufbaus des Gesundheitsschutzes in der DDR

Alexander Mette 


\title{
Die Bedeutung der sowjetischen Neuropathologie und Psychiatrie für die Neugestaltung der Fachrichtung während des Aufbaus des Gesundheitsschutzes in der DDR*
}

\author{
Von ALEXANDER METTE (Berlin)
}

Bei der Berührung mit Forschungsmethoden und -ergebnissen sowjetischer Wissenschaftler sind auch für die Vertreter einzelner Sparten der Medizin bekanntlich Anregungen mit nachhaltiger Wirkung entstanden. In hervorragendem Maße betraf das die Sozialhygiene, speziell die Organisation des aktiven Gesundheitsschutzes. Es verging allerdings geraume Zeit, bis die Gesichtspunkte, die N. A. Semasko (1874-1949), der erste Volkskommissar für das Gesundheitswesen, entwickelte, für andere Länder Aktualität erlangten, weil dazu politische Vorbedingungen erforderlich waren. Unabhängig davon boten die neuen Theorien und Maßnahmen hier für fortschrittliche Kollegen jedoch willkommenes Material zur Orientierung, und ihr Einfluß auf einzelne deutsche Ärzte und auf das Gesundheitsprogramm der KP.D ist demgemäß in den 20er Jahren auch bereits erkennbar geworden $[1,2]$.

Daß auch im Blickfeld der Neuropathologen und Psychiater einiges Neue sichtbar wurde, hängt mit der Eigenart bestimmter Aufgaben des Faches zusammen. Das Bestreben, Kranken, die bei der Behandlung und Pflege ihrem beruflichen und häuslichen Leben oft lange entzogen wurden, soviel Bewegungsfreiheit und Betätigung wie irgend möglich zu verschaffen, führte zu Umgestaltungen im Gepräge ihrer Unterbringung, die Anlaß zu der Feststellung gaben, daß man, ,die neueZeit der Sowjets" auch in psychiatrischen Krankenhäusern spüre [3]. Was sich hier als neuer Stil herausbildete, übertraf die von humanitären Absichten geleiteten besten Beispiele fortschrittlicher Geisteskrankenbetreuung der Vergangenheit - bei Wahrung aller guten Komponenten, die ihnen eigen waren - durch das typische Entstehen beweglicher Kollektive von Ärzten, Sanitätern und Patienten, welche zum Hebel für den therapeutischen Erfolg werden konnten.

In der russischen Neuropathologie und Psychiatrie hatte im 19. Jahrhundert bei den bedeutenden Repräsentanten bereits ein enges Verhältnis zur pathologischen Anatomie und zur Physiologie überwogen. A.Ja. Koミevnikov (1836-1902) und

* Frau Obermedizinalrat Dr. med. Hette Lammert in Verehrung gewidmet.

8* 
S. S. Korsakov (1854-1900), V. M. Kernig (1840-1917) und V. P. Serbskij (1855 bis 1917) boten dafür - mit vielen anderen - eine Fülle von Belegen. Der Begründer der modernen Physiologie in Rußland. I. M. Seienov (1829-1905), der mit den revolutionären Demokraten der 60er Jahre in die Arena trat, machte - wie vor ihm theoretisch bereits $W$. Griesinger $(1817-1868)[4,5]$ - auf die umfassende Rolle der reflektorischen Vorgänge im Zentralnervensystem aufmerksam und war damit der Wegbereiter für die Forschungen I. P. Pavlovs (1849-1936) und für das spezielle Studium der Innervationsprozesse durch $N$. E. Vvedenskij (1852-1922) und A. A. Uchtomskij (1875-1942) geworden [6]. International so bekannte Neurologen und Psychiater wie V.M. Bechterev (1857-1927) und G. I. Rossolimo (1860 bis 1928) bzw. P. B. Gannuskin (1875-1933) und V. A. Giljarovskij (1875-1959) bewegten sich in den Bahnen der Tradition. Gannuskin, der bei der Psychopathie Umweltfaktoren hohes Gewicht beilegte, und Giljarovskij, der einen Modus der Neurosenbehandlung in kollektivem Zusammenwirken propagierte, waren beide Schüler Korsakovs gewesen. Sie standen mit ausländischen Autoren in ziemlich regem Kontakt und werteten zeitweise die Errungenschaften der russischen und der sowjetischen Neurophysiologie nicht in dem Umfang aus wie Kliniker, die vorerst bei Pavlov gearbeitet hatten - zum Beispiel N. I. Krasnogorskij (1882 bis 1961), der bekannte Pädiater, M.K. Petrova (1874-1948) und der Psychiater A. G. Ivanov-Smolenskij (geb. 1895).

Pavlov selbst hatten seine um die Jahrhundertwende begonnenen Studien über die Tätigkeit der Großhirnhemisphären, insbesondere der Großhirnrinde, für Probleme der Psychiatrie empfänglich gemacht, und seit 1918 nutzte er Gelegenheiten zur Beschäftigung mit Patienten in Pflegeanstalten für Geisteskranke zur Vertiefung seiner psychiatrischen Kenntnisse und widmete sich ihnen immer eingehender. Ein Vortrag, den er nach den ersten konkreten Beobachtungen hielt, erschien 1919 und erhöhte das Interesse der Fachkliniker für seine Lehre von der höheren Nerventätigkeit. Er enthielt schon den Hinweis auf die bei psychotisch Kranken häufig anzutreffende umfangreiche Hemmung, der den Keim zu der Konzeption von der Schutzhemmung bildete, die er im Sinne einer durch therapeutische Methoden unterstützbare Selbststeuerung der Rindenzellen ausbaute. Bevor er elf Jahre später erneut über eine ,,Abschweifung in das Gebiet der Psychiatrie" berichtete, folgten als Arbeiten, die benachbarte Themen behandelten, seine Mitteilung „Über die sogenannte Hypnose der Tiere“ (1921), der Artikel „,Die Beziehungen zwischen Erregung und Hemmung, sowie experimentelle Neurosen an Hunden", der zum Teil auf Untersuchungen von Frau Petrova fußte (1925), und der Beitrag ,,Die physiologische Lehre von den Typen des Nervensystems oder den Temperamenten" (1928). Ihre internationale Resonanz war bei dem Ansehen, das der Autor genoß, groß, und das galt auch von den anschließend veröffentlichten weiteren Arbeiten, die diesen Themenkreis tangierten und die „Phasen“ verringerter Arbeitsfähigkeit der Rindenzellen, Neurosen des Menschen und des Tieres, das physiologische Verständnis der Symptomatologie der Hysterie, die physiologische Erklärung der Zwangsneurose und der Paranoia und die allgemeinen 
Typen der höchsten Nerventätigkeiten der Tiere und des Menschen behandelten [7]. Sie wurden in den Fachorganen anderer Länder rasch rezensiert und erschienen auch im vollen Text in deutscher oder französischer Übersetzung im Ausland [8]. Ihrer Verbreitung kam der Umstand zugute, daß Pavlov Nobelpreisträger war, außerdem aber auch die Förderung, die der Sowjetstaat seinem Wirken und dem seiner Schüler seit der von Lenin unterzeichneten Sonderverordnung aus dem Jahre 1921 angedeihen ließ.

Einer knappen Formulierung über die Bedeutung des neuen Weges, den er einschlug, um die Gesetzmäßigkeiten der Hirntätigkeit zu erschließen, begegnet man in einer Diskussion Pavlovs mit Bechterev im Jahre 1908 - ein Jahr vor seinem viel beachteten Vortrag ,Naturwissenschaft und Gehirn“. ,Als Naturwissenschaftler“ - heißt es hier - ,kann und darf der Physiologe nicht die frühere subjektive Methode der Analogie mit seinen Gefühlen und Qualitäten verwenden; ihm bleibt ein anderer Weg: Er muß objektiv die Verbindung zwischen den verschiedenen äußeren Reizen und der Tätigkeit der Hirnrinde studieren. In dieser Beziehung aber ist in den letzten 30 Jahren rein gar nichts getan worden, an diese Frage ist man überhaupt nicht herangetreten. Die Methode der bedingten Reflexe aber gibt uns gerade diese Möglichkeit; mit ihrer Hilfe ist bereits ein gewaltiges System neuer Tatsachen entdeckt worden ..." [9]. Daß er diesem Prinzip konsequent treu blieb, trug ihm die hohe Wertschätzung jener Wissenschaftler seines Landes ein, die über die Gefahr der Verfälschung unzureichend gesicherter Abstraktionen durch spekulative. der konventionellen Ideologie des Bürgertums entsprechende Deutungen Klarheit gewonnen hatten. Er ließ sich unbeirrt von ihm leiten, auch als er 1932 in weiterem Sinne als zuvor beim Menschen zwischen dem ,ersten Signalsystem“ (dem alleinigen im Tierorganismus sonst) und dem ,,zweiten“ (der Sprachtätigkeit im umfassenden spezifisch menschlichen Betracht) unterschied und sich zwei Jahre später in einer Vorlesung ausdrücklich mit der experimentellen Pathologie der höheren Nerventätigkeit beschäftigte. Sein letzter Vortrag im Ausland, in dem er unter Bezugnahme auf seine Kennzeichnungen der allgemeinen und der speziell menschlichen Typen der höheren Nerventätigkeit auf den physiologischen Mechanismus der neurotischen und psychotischen Symptome einging (2. internationaler Neurologenkongreß in London, 1935), zeigte, daß das Bemühen um überzeugende Ergänzungen zu den Resultaten der Laboratoriumsarbeit jenseits von deren Grenzen Kenntnisse erbracht hatte, durch die das Verständnis zahlreicher bekannter Symptome vertieft und dem Gedanken an neue therapeutische Möglichkeiten eine Stütze gegeben wurde.

Die Ansichten, zu denen Pavlov im Bereich von Erwägungen gelangte, die für die psychiatrische Klinik fruchtbar zu werden versprachen, reiften langsam. Von entscheidender Bedeutung war es, daß die präzise Beobachtung der bedingt-reflektorischen Vorgänge ihn und seine Schüler aus der psycho-morphologischen Betrachtungsweise hinausführte und ein Bild von der Tätigkeit der Hirnrinde vermittelte, das die von der Mehrzahl der Hirnpathologen anerkannten Auffassungen an Weite und Genauigkeit übertraf. In der Richtung, die Sečenov beschritten hatte, 
und im Einklang mit den neurophysiologischen Grundanschauungen Korsakovs bemühten sich Pavlov, Vvedenskij und Uchtomskij nebst ihren Schülern um die Klärung der Dynamik der Erregungs- und der Hemmungsprozesse wie um deren Erscheinungsformen, die im allgemeinen nahezu gänzlich vernachlässigt wurden. Pavlovs Abgrenzung der ,,ausgleichenden“, der ,,paradoxen“ und der ,,ultraparadoxen" Phase beim Übergang vom Wachzustand zum Schlaf, Vvedenskijs ..Parabiose" und Uchtomskijs ,Dominante" $[10,11]$ verhalfen in der Pathophysiologie zu überaus wertvollen Anhaltspunkten. Von großem Gewicht waren auch Pavlovs Ermittlungen über die Physiologie des Schlafes, die Verwandtschaft von Schlaf und Hypnose, die Schutzhemmung und ihre bedeutsame Rolle als Bestandteil von Krankheitsprozessen und ihrer Symptomatologie - wie endlich die im Tierexperiment erhärtete Entstehung von Neurosen infolge Belastung der Großhirnrindentätigkeit mit gewissen, ihre Leistungsfähigkeit überfordernden Aufgaben.

Während das Echo des Auslandes auf den pathophysiologischen Ertrag der Lehre vom bedingten Reflex und auf die mit ihr verknüpfte Analyse der Befunde in einer Reihe von klinischen Fächern recht lebhaft, in der Pädiatrie sogar stark [12], war, erwiesen gerade Neurologie und Psychiatrie sich als weniger aufnahmebereit. Die Psychiatrie gehört zu den Fächern, in deren Schrifttum der Gegensatz zwischen dem spätbürgerlichen Denken und dem dialektisch-materialistischen, das in der Sowjetunion vorherrschte, deutlich offenbar wurde, und daraus ergaben sich Hindernisse für die Verbreitung der sowjetischen Materialien in diesem Wissenschaftszweig.

In Deutschland stand die Psychiatrie im 3. Jahrzehnt unseres Jahrhunderts im Zeichen ungeklärter Widersprüche, die ein Nebeneinander unterschiedlicher Strömungen bedingten. Mit am eindrucksvollsten legten das Referate von $O$. Bumke (1877-1950) und K. Kleist (1879-1960) auf einer gemeinsamen Sitzung des deutschen Vereins für Psychiatrie und der Gesellschaft Deutscher Nervenärzte 1924 in Innsbruck an den Tag $[13,14]$.

Beide wiesen auf die Lockerung der Beziehungen zwischen klinischer Psychiatrie und Hirnpathologie nach dem Tode Th. Meynerts (1833-1892) und K. Wernickes (1848-1905) hin, illustrierten sie am weiteren Werdegang des Faches, bewerteten sie jedoch grundverschieden. Unter den bedeutenden Klinikern hatte E. Kraepelin (1856-1925) W. Wundt (1832-1920) nachhaltiges Interesse gewidmet, die Pflege der Pathohistologie fortgesetzt, sich aber hauptsächlich dem Studium der Verlaufsformen der endogenen Psychosen zugewandt. Seinen von K. Kahlbaum (1828-1899) übernommenen Begriff der ,,Krankheitseinheit“ kritisierten A.E. Hoche (1865-1943), K. Bonhoeffer (1868-1948) und bedingt auch Bumke. Den genealogischen Aspekt E. Rüdins (1874-1952), des langjährigen Mitarbeiters von Kraepelin, und die konstitutionsbiologische Klassifizierung E. Kretschmers (1888-1964), für die die Aufgliederung Kraepelins richtungsweisend gewirkt hatte, betrachteten Bumke und Kleist hingegen als Bereicherung, obwohl der Entwertung der äußeren Noxe durch sie Vorschub geleistet wurde. Bumke 
stimmte auch den Versuchen zu, die K. Jaspers (geb. 1883) und $H$. W. Gruhle (1880-1963) - angeregt durch die Schriften W. Diltheys (1833-1911) und M.Webers (1864-1920) - unternahmen, um die Gesichtspunkte der ,,verstehenden Psychologie" in der Psychiatrie anzuwenden. Kleist sah in der Anlehnung an die ,Lebensphilosophie“ und die neuerstandene Psychologie, die sich vorwiegend mit den intentionalen Vorgängen beschäftigte und einer Überschätzung des Wertes der Selbstbeobachtung die Tür öffnete, einen Rückzug. Jaspers, Gruhle, Kurt Schneider (geb. 1887), wie ebenfalls Bumke, hielten die Bemühungen der Histopathologen in Hinblick auf die klinische Psychiatrie nicht für sonderlich verheißungsvoll. Schneider erhob sogar die Forderung nach einer ,,reinen“, von der ,,neurologischen Knechtschaft" befreiten Psychiatrie und hat sich später Konzeptionen genähert, die in das Denkgebäude der Scholastik passen [15]. Kleist wiederum erwartete von den Resultaten des Histopathologen neue Aufschlüsse und betonte auch, daß die bedingten Reflexe Pavlovs deutlichere Einblicke ermöglichten als die früheren Vorstellungen über Erregungsleitung, Hemmung und Bahnung. Ungeteilte Anerkennung fand die Leistung J. Wagner von Jaureggs (1857-1940), und als hoch beachtlich wurden zudem die Erfahrungen bewertet, die bei der Enzephalitisepidemie gesammelt worden waren, nachdem das Krankheitsbild der Encephalitis lethargica im gleichen Jahre 1917 von $C$. Economo (1876-1931) in Wien beschrieben worden war, indem Wagner von .Jauregg dort die erste therapeutische Infektion mit Malaria wagte.

Man hat die Verringerung des Interesses an der Histopathologie und der Neurophysiologie bei namhaften Psychiatern um die letzte Jahrhundertwende und im ersten Viertel unseres Jahrhunderts durch einen Mangel an weiteren aufschlußreichen Resultaten in diesen Forschungsbereichen veranlaßt geglaubt. Bei genauerer Rekapitulation ergibt sich jedoch ein etwas anderes Bild. Als Kraepelin sein Hauptaugenmerk auf den Krankheitsverlauf richtete und als in der Psychologie die Konzeption Diltheys auftauchte und damit der Weg angebahnt wurde, den Jaspers und Gruhle hernach beschritten, das heißt Mitte der 90er Jahre, wartete beispielsweise auf dem 3. Physiologenkongreß in Bern Vvedenskij mit Forschungsergebnissen über das neurale Optimum und Pessimum auf [16], an die anzuknüpfen sich gelohnt haben würde. Daß die Neurologen und Psychiater der westlichen Länder in der Mehrheit kaum davon Notiz nahmen, war dem Umstand mit zuzuschreiben, daß ihre Empfänglichkeit für Methoden und Fakten, die den Stempel der materialistischen, demokratisch-humanistischen Haltung Sečenovs [17] trugen, durch den Einfluß der imperialistischen Ideologie getrübt wurde, die seit dem Übergang zum Monopolkapitalismus in den führenden Industriestaaten in breiten Kreisen des Bürgertums Oberhand gewann. Agnostizistischen Konjekturen, denen zuvor schon vermehrt gehuldigt wurde, traten in dieser Phase bei Medizinern immer häufiger irrationalistische Spekulationen an die Seite. Namhafte Naturwissenschaftler fühlten sich aus übertriebener Sorge vor vermeintlichen rationalistischen Fangschlüssen dazu bewogen, mit Unklarheiten vorlieb zu nehmen. Dafür war auch S. Freuds (1856-1939) Wendung von der Neuropathologie 
zu der von $J$. Breuer (1842-1925) und ihm in Wien begründeten ,,psychokathartischen Methode" ein Beispiel, die durch ihre gemeinsamen Veröffentlichungen 1893 und 1895 evident wurde [18].

Seine Psychoanalyse wäre ohne die sexualpathologischen Standardwerke, die vorlagen, obenan das von R. von Krafft-Ebing (1840-1902), und ohne die Wirkung, die gegen Ende des Jahrhunderts von Fr. Nietzsches (1844-1900) Schriften auszugehen begann, vermutlich länger ohne große Resonanz geblieben. Die Überbewertung der Libido, die sich in ihren pathogenetischen, typologisch-charakterologischen und therapeutischen Thesen abzeichnete, stellte eine verwegene und für manche attraktive - Steigerung und Zuspitzung der Tendenz dar, dem Erleben bis zu verborgenen Quellen nachzuspüren. In diesem Bestreben gingen die Freudsche Tiefenpsychologie und die Abzweigungen A. Adlers (1870-1937) und C.G.Jungs (1875-1961) weiter als die Bemühungen phänomenologischer Observanz. Der Einfluß, der von Dilthey, Nietzsche und anderen Repräsentanten der Lebensphilosophie wie G. Simmel (1858-1918), H. Bergson (1859-1941) oder L. Klages (1872-1956) auf die Psychiatrie in Deutschland ausging, läßt sich dem „Handbuch der Geisteskrankheiten“ [19] entnehmen, das Bumke in den 30er Jahren herausgab. Dieser selbst kritisierte zwar - wie es auch Jaspers tat - die Dogmen Freuds, suchte als kompromißfreundlicher Eklektiker auf dem Boden dualistischer Anschauungen, der er war und blieb, im übrigen aber alles zu vereinigen, was er zuvor schon bejaht hatte und was an Kommensurablem inzwischen hinzugekommen war.

Der Umstand, daß die Mehrzahl der Lehrstuhlinhaber des Fachgebiets in der Weimarer Republik der Freudschen Psychoanalyse keine Geltung zubilligten, aber am Institut der deutschen Zweigorganisation der Internationalen Psychoanalytischen Vereinigung Gelegenheit zur Ausbildung bestand, verschaffte ihr in Kreisen, die sezessionistischen Bewegungen geneigt waren, etwas wie Nimbus. Auch fortschrittliche und mit dem Marxismus sympathisierende junge Mediziner und Pädagogen brachten ihr Interesse entgegen und erlernten die Methode. 1926 erschien in der von Freud herausgegebenen „Internationalen Zeitschrift für Psychoanalyse" ein Beitrag des sowjetischen Fachkollegen A. R. Lurija, der bekannte Forschungsergebnisse Pavlovs, Vedenskijs und Uchtomskijs mit gewissen Konzeptionen Freuds verglich [20] und starke Beachtung fand. Bald nachdem V. I. Lenins „Materialismus und Empiriokritizismus" 1927 endlich in autorisierter deutscher Übersetzung vorlag, entspannen sich Auseinandersetzungen über die Frage der Vereinbarkeit Freudscher Auffassungen mit marxistischen. Sie beschäftigte neben S. Bernfeld (geb. 1892) und W. Reich (geb. 1897) eine Reihe der jüngeren Fachkollegen, die sich in Wort und Schrift [21] an der Debatte beteiligten, sehr intensiv. Als I. Sapir daraufhin in der Zeitschrift „Unter dem Banner des Marxismus" [22] zeigte, daß Freuds Behauptungen trotz manches im einzelnen Gültigen der Kritik seitens der dialektisch-materialistischen Lehre nicht standhielten, die Überschätzung der Wirkung biologisch-instinktiver Kräfte jedoch die Gefahr des Absinkens in einen gesellschaftlich-historischen Idealismus herauf- 
beschwöre, wurde das nicht prinzipiell bestritten. Die Befürworter des Gedankens an eine Annäherung glaubten aber einwenden zu können, daß man biologistischen Ingredienzien bei Kraepelin, Rüdin und Kretschmer ebenso unverkennbar begegne wie bei Freud, bei Jaspers und Gruhle - in Anlehnung an die Soziologie Max Webers [23] - wiederum in gleichem Maße Elementen einer idealistischen Geschichtsinterpretation. Als Wahrheitsgehalt steckte in diesem fehlgehendenVerteidigungsversuch freilich nur die Tatsache, daß die unterschiedlichen Richtungen inDeutschland durchweg nicht sehr weit voneinander differierten, weil sich, bei der einen wie der andern, in ihrem Bild eines aus den realen Beziehungen herausgelösten Menschen Einflüsse von Spielarten der imperialistischen Ideologie manifestierten. Zweifellos war es diesem Umstand auch zuzuschreiben, daß die einheitliche naturwissenschaftliche und gesellschaftswissenschaftliche Erschließung der menschlichen Wirklichkeit, auf die es ankam und die vom marxistischen Standpunkt aus erreichbar war, jeweils unterblieb.

Vier Jahre bevor dann die reaktionärsten und brutalsten Vertreter des deutschen Monopolkapitalismus und Militarismus im Staate an die Macht kamen, veröffentlichte $H$. Berger (1873-1941) die erste seiner Mitteilungen über das EEG des Menschen [24] und bereicherte die Diagnostik und die Forschung dadurch mit einer sehr leistungsfähigen Methode. Bis ihre Tragfähigkeit sich ausgiebiger nachprüfen ließ, traten für die Psychiatrie unter dem fortschrittsfeindlichen Hitlerregime Bedingungen ein, die die fruchtbare Anwendung der wertvollen Entdeckung in Klinik und Laboratorium stark beeinträchtigten. Auf ausländischen internationalen Kongressen hat Berger sie Foren, die für sie kompetent waren, nur zweimal im Jahre 1937 näherbringen können. Obwohl es in der Sowjetunion verhältnismäßig bald zur Einführung in die Laboratoriumsarbeit kam, zog die Auswertung der Ergebnisse in Hinblick auf den Schließungsmechanismus des bedingten Reflexes sich bei den später im internationalen Maßstab daran arbeitenden Forschern lange hin und ist sogar auch in diesem Jahrzehnt noch im Gange [25].

Den Nazimachthabern und ihren Hintermännern bot der therapeutische Nihilismus, der trotz der Veränderung des Bildes der Kliniken durch die Erfolge der Malariakur hinsichtlich der endogenen Psychosen in der Psychiatrie der kapitalistischen Länder überwiegend fortbestand, Anlaß zu gesetzlichen Maßnahmen im Rahmen der Medizin, die in der Handhabung an die ihrer verbrecherischen Rassenpolitik erinnerten und schließlich zur systematischen Ermordung psychotisch Kranker überleiteten. Als Mitte der 30er Jahre Berichte über die Ergebnisse der Kampfer-, der Insulin- und der Elektrokrampfkur es als empfehlenswert erscheinen ließen, die Behandlung damit aufzunehmen, schuf das in ihrem Machtbereich keinen Wandel, da die Schlußfolgerungen aus den tendenziösen Behauptungen über die Heredität und den schicksalsmäßigen Verlauf aufrechterhalten wurden.

Für die Propagierung konstitutionsbiologischer Thesen, die den Wünschen des Regimès ausdrücklich entgegenkamen, erwiesen sich Darstellungen wie die des Psychologen E.R.Jaensch (1883-1940) als dienlich [26]. In der ,Tiefenpsychologie“" 
mußten die Freudschen Konzeptionen den mystischer Verbrämung weniger abholden von Jung weichen, und ihre literarische Vertretung erforderte große Geschicklichkeit, wenn sie nicht durch Beschlagnahme beantwortet werden sollte wie schon sehr bald eine Schrift des Verfassers [27]. Auch von Jaspers wurde die Aufmerksamkeit abgelenkt, während der Philosoph M.Heidegger (geb. 1889) im Gesichtsfeld der Psychopathologen und Psychotherapeuten unbehindert breiteren Raum einnahm. Zu den mit Mißtrauen Beobachteten gehörte auch der Hirnforscher $O$. Vogt (1870-1959), nachdem er etliche Jahre in der Sowjetunion wirkte. Übersetzungen von Schriften sowjetischer Autoren begegnete man nur noch ganz vereinzelt und bald gar nicht mehr. Das betraf unter anderem die letzten Arbeiten Pavlovs. Auch von K. M. Bykov (1886-1959) erschien keine Übersetzung mehr in Deutschland, und ebenso wurde die Veröffentlichung von $A$. D. Speranskijs (1888 bis 1961) international viel beachtetem Buch ,Grundlagen der Theorie der Medizin" [28] von den Nazis vereitelt. Referate über Fortschritte der experimentellen und klinischen Forschung in der Sowjetunion, die immer noch auftauchten, blieben, den Verhältnissen entsprechend, ohne gebührende Resonanz.

Der Zeitabschnitt, in dem diese Schranken bestanden, war in der sowjetischen Medizin durch vielseitige Bemühungen um neue Erfolge gekennzeichnet. Fortschrittliche therapeutische Verfahren wie die Arbeitstherapie bei Schizophrenen und die Möglichkeiten ihrer ambulanten Betreuung in gegebenen Fällen wurden erläutert, so von V.P. Osipov (1871-1947), der auch in Hinblick auf die Abgrenzung der Krankheitsbilder - ähnlich wie Giljarovskij - Vorschläge machte und stärkere Berücksichtigung ätiologischer Komponenten empfahl. Psychischen Störungen bei hepatolentikulärer Degeneration widmete sich $N$. V. Konovalov (1900-1966). Pavlov griff die Diskussion über aktuelle Fragen in der Psychiatrie nach seiner Hysterieschrift noch eifriger auf und trug wiederholt erneut seine Auffassungen vom 1. und 2. Signalsystem, von den Typen der höheren Nerventätigkeit und von der Nosologie und Symptomatik der Neurosen und Psychosen in Fachkreisen vor. Nächst ihm beteiligten Petrova, Ivanov-Smolenskij, E. A. Popov (1899-1961) und auch Krasnogorskij sich daran. Wie Pavlov seine Feststellungen über das Zustandekommen von Induktionsvorgängen und von Phasenveränderungen für das Verständnis pathodynamisch schwer analysierbarer klinischer Erscheinungen fruchtbar zu machen versuchte, zeigt sein offener Brief an P. Janet (1859-1947), in dem er auch den Vorwurf, mechanistisch zu verfahren, zurückwies [29]. Zur Deutung von Symptomen bei Paranoia zog er außer dem eigentümlichen Gepräge der Phasenzustände auch Beobachtungen über Trägheit des Erregungsprozesses und des Hemmungsprozesses mit heran [30]. Nachdrücklich setzte er sich für den Ausbau eines Regimes in psychiatrischen Krankenanstalten ein, das der nervalen Anfälligkeit der Patienten in vollem Umfang Rechnung trug [31], und einhelliger ließen sich aus der Bedeutung, die er der Schutzhemmung in gewissen stuporösen Zuständen beilegte, nun auch Erwartungen für die Therapie ableiten. 
Nach Pavlovs Tod nahm die angebahnte Entwicklung ihren Fortgang. Schon seit 1934 zog Ivanov-Smolenskij aus Untersuchungen von Kranken mittels des von ihm bereits zuvor benutzten, äußerlich an die gebräuchlichen Assoziationsproben erinnernden Wortexperiments Rückschlüsse auf die Besonderheit der Wechselwirkung der Signalsysteme unter den vorliegenden pathologischen Bedingungen [32]. 1933 war von ihm ein Buch über die ,Grundprobleme der Pathophysiologie der höheren Nerventätigkeit" mit einem Vorwort von Pavlov erschienen [33]. Den Leitsatz, möglichst im Sinne der Schutzhemmung zu behandeln, vertrat er mit großer Konsequenz. Von der Dauernarkose mit der anfangs als Medikation übernommenen Cloettaschen Mischung [34] ging man, harmlosere Mittel bevorzugend, zur Dauerschlafbehandlung und zur Kur mit prolongiertem Schlaf über. Auch die Kombination mit Insulin- oder Cardiazolinjektionen in kurmäßiger Verabreichung, bei der eine Minderung der Zahl der Insulin- bzw. Cardiazolgaben erwirkt wurde, kam in Gebrauch. Die Konvulsionstherapie, die in den westlichen Ländern der 1922 von J.Klaesi (geb. 1883) angegebenen Dauerschlafbehandlung gegenüber zur Methode der Wahl wurde [35], fand in der UdSSR in den allgemein üblich gewordenen Formen nur vorübergehend uneingeschränkten Anklang, weil die nachteiligen Begleiterscheinungen, die ihr oft anhafteten, nicht übersehen wurden. Je sicherer es gelang, die hier entwickelteArt der Schlaftherapie gefahrlos und schonend durchzuführen, desto mehr wurde sie als via regia empfohlen. Eine der neuen Aufgabenstellung entsprechende, mehrere Komponenten in umsichtiger Weise vereinigende Schutztherapie baute als einer der ersten V.P. Protopopov (1880-1957) in seiner Klinik in Charkov aus.

Umfangreiche Erfahrungen bei Traumen wurden unter Berücksichtigung der pathogenetischen und therapeutischen Auffassungen, die Pavlov vertreten hatte, im Großen Vaterländischen Krieg gesammelt. Man beobachtete die Symptomatik der Schutzhemmung bei Explosionswirkungen, bei Luftkontusion, bei zahlreichen Kopfverletzungen und anderen Verwundungen. Mit der Schlaftherapie bei Traumen im Fachbereich beschäftigten sich Ivanov-Smolenskij, E. A. Asratjan (geb. 1903) und andere [36]. Dabei wurden beachtliche Wiederherstellungsmöglichkeiten unter Beweis gestellt [37]. In allen Einzelzügen tritt, wie man leicht ersieht, das Streben nach höchster Entfaltung der Hilfsmaßnahmen unter weitestgehender Vermeidung in höherem Grade peinvoller Verfahren zutage, das für die humanitären Prinzipien des sowjetischen Gesundheitsschutzes typisch ist. Parallel dazu begann man mit der Bekämpfung des ,,klinischen Todes“, mit Erwägungen über einen gangbaren Weg zur Schmerzausschaltung unter der Geburt und der feineren Analyse des Krankheitsbeginns in unterschiedlichen Fachbereichen, auf die sich dann die Dispensairebetreuung stützen konnte [38]. Pavlovs Begriff der Plastizität der höheren Nerventätigkeit und die drei Prinzipien, die er seiner Reflextheorie zugrunde legte, das des Determinismus, das der Analyse und Synthese und das der Strukturiertheit, machten die sowjetische Psychiatrie, soweit sie ihm und seinen Schülern folgte, zu einem Bollwerk gegen metaphysische, biologistische und fatalistische Spekulationen und dienten der Verbreitung der dialektischmaterialistischen Abbildtheorie im Fachbereich. 
Als fortschrittliche Psychiater und Neurologen in Deutschland nach der Befreiung rom Hitlerfaschismus durch die siegreiche Sowjetunion und ihre Verbündeten wieder unbehindert arbeiten konnten, wurde ihnen - vor allem in der damaligen sowjetischen Besatzungszone - die Möglichkeit geboten, die zunächst vorhandene Kluft durch Aneignung der benötigten Kenntnisse zu überbrücken. Die erschreckenden Berichte über die während des Hitlerregimes von Medizinern verübten grauenhaften Verbrechen an Geisteskranken und anderen hilflosen Menschen schärften die Kritik an den Mängeln und Schwächen der konventionellen ärztlichen Anschauungen - insbesondere an den verbreiteten Erblichkeitsdogmen, dem konstitutionsbiologischen Prädestinationsglauben und dem therapeutischen Nihilismus.

Etwas näher mit der sowjetischen Medizin vertraute Kliniker wie der bekannte Internist $W . H$. Veil (1884-1946) hoben die Bedeutung der exogenen Noxe für alle Fachbereiche mit Nachdruck hervor [39], und dieser unterzog in der 1946 erschienenen erweiterten Fassung seiner bekannten Studie über Goethe auch die Hereditäts- und Periodizitätsschemata in den Goethe betreffenden Darlegungen E. Krelschmers einer grundsätzlichen Kritik [40].

Auf der ersten wissenschaftlichen Tagung der Psychiater und Neurologen in der damaligen Sowjetischen Besatzungszone Deutschlands im Mai 1948 wurde eine Resolution angenommen, die der Empörung der Anwesenden über die Naziverbrechen Ausdruck verlieh. Auch sonst war der Vorsatz, der Menschlichkeit wieder Genüge zu tun, dominierend. H. Schwarz, Greifswald (geb. 1898), der die ärztliche Tätigkeit im sogenannten ,Erbgesundheitsverfahren": kurz darauf in einer Monographie grundlegend kritisierte [41], forderte genaue Indikationsfindung für die Elektroschockbehandlung und empfahl die Ablehnung der präfrontalen Leukotomie aus dem gleichen Motiv, das 1950) in der Sowjetunion zu ihrem Ver. bot veranlaßte. Obwohl bereits ein Jahr zuvor ein Beitrag von Asratjan in deutscher Übersetzung veröffentlicht worden war [42], widmete sich nur ein einziges Referat ausführlich einem sowjetischen Autor, nämlich das von $K$. R. von Roques (geb. 1897) über die Pathologie Speranskijs. Eindrucksvolle Fakten enthielt ein Bericht über die Entwicklung der psychiatrischen Fürsorge in der damaligen SBZD, der $\mathrm{zu}$ tröstlichen Hoffnungen berechtigte. In mehreren Referaten wurde von alten Vertretern der Freudschen Richtung für die Anerkennung der Psychoanalyse geworben. Der Verfasser suchte in einem Referat zwischen Konzeptionen Bernfelds und den in Makarenkos (1888-1939),,Weg ins Leben“ niedergelegten Erfahrungen eine Brücke zu schlagen [43].

Die Erwartungen auf offizielle Anerkennung der Psychoanalyse, die damals bei manchen alten Freudianern bestanden, wurden nur partiell und nicht auf die Dauer erfüllt. Es zeigte sich - vom Verfasser schon bald erkannt und hervorgehoben $[44,45]$ - daß nicht wenige Psychiater und Neurologen, die Freuds Anschauungen und Methoden vor und während der Zeit des Hitlerregimes abgelehnt und teils nach Kräften bekämpft hatten, umschwenkten, als sie mit deren Bejahung nunmehr gegen den Marxismus frondieren zu können vermeinten und in einer 
Verquickung mit dem Existentialismus oder mit dem Neothomismus das richtige Mittel dafür zu finden glaubten. Auseinandersetzungen darüber nahmen auch in der $1949 \mathrm{im}$ 1. Jahrgang erschienenen, vom Verfasser herausgegebenen Zeitschrift „Psychiatrie, Neurologie und medizinische Psychologie“ anfangs ziemlich viel Raum ein [46]. Die 100. Wiederkehr von Pavlovs Geburtstag im September 1949 rückte die Bedeutung seiner Lehre für die Medizin und speziell auch für die Psychiatrie und Neurologie dann jedoch nun ebenfalls in Deutschland stark in den Brennpunkt. Sowohl das neue Fachorgan als auch die von $K$. Winter (geb.1910) herausgegebene ,Zeitschrift für ärztliche Fortbildung“ brachten ausführlichere Würdigungen des großen Forschers, und in etlichen anderen begegnete man Hinweisen, die in erster Linie des Nobelpreisträgers gedachten. Im gleichen Jahr begann der Internist R. Baumann (geb. 1911) die Arbeit mit der Schlaftherapie und entwickelte damit den Ansatz zu dem von ihm geleiteten Institut für kortikoviszerale Pathologie und Therapie in Berlin-Buch [47]. Der Psychiater und Neurologe G. Göllnitz, Rostock (geb. 1920) schloß sich der Bewertung motorischer Ausfallserscheinungen an, die $N . I$. Ozereckij (1893-1955) mit seiner metrischen Untersuchungsmethode in die Psychiatrie der Kinder und Jugendlichen eingeführt hatte, und widmete sich der durch sie gewährleisteten Verfeinerung der Diagnostik [48].

Die Fühlung mit dem sowjetischen Fachschrifttum und einzelnen der hervorragenden Autoren erfuhr nach der Gründung der Deutschen Demokratischen Repuklik im Laufe weniger Jahre rasch weitere Belebung und Vertiefung. Entscheidend gefördert wurden die Bindungen durch die Veröffentlichung des Gesamtwerks von Pavlov in sorgfältiger, von L. Pickenhain (geb. 1920) verantwortlich geleiteter Übersetzung und das Erscheinen wichtiger anderer übersetzter Publikationen sowjetischer Physiologen, Neuropathologen und Psychiater [49]. Ein kurzer Bericht des Verfassers, der sich auf diese Jahre bezog, erschien 1957 im ,Korsakow-Journal für Neuropathologie und Psychiatrie" [50]. Breiter auf dieFachhistorik in Deutschland eingehend, widmete sich ihnen der bekannte sowjetische Psychiater $G . K$. $U \dot{s} a k o v$ (geb. 1921) in einer umfangreicheren, die Details ausführlicher berücksichtigenden Darstellung [51]. Auf die Hauptzüge des Themas, das mehrere der persönlich Beteiligten wiederholt literarisch beschäftigt hat [52], lenkten auch Stellungnahmen aus philosophischer Sicht mehrfach den Blick [53].

In der Rückschau erscheint eine Art Affinität zur Lehre von der höheren Nerventätigkeit bei psychotherapeutisch stärker interessierten Kollegen als charakteristischer Zug. Neben einer Reihe von Physiologen, Pädiatern, Sozialhygienikern und Internisten bildeten gerade mit der Theorie und Praxis der Psychotherapie ausgiebiger Vertraute - so D. Müller-Hegemann, Leipzig (geb. 1911) und der Verfasser - im Fachbereich die Gruppe der Aufnahmebereitesten und Empfänglichsten. Die Gepflogenheit, in der Kasuistik scheinbar unbedeutenden Faktoren gebührendes Gewicht beizulegen, half dabei mit. Andererseits übten die Hypnoseund Neurosestudien der Pavlov-Schule sowie die Methoden der Arbeitstherapie und der Behandlungskollektive auf psychotherapeutisch Bewanderte starke Anziehungskraft aus. Schon vor der unmittelbaren Bekanntschaft mit dem jüngsten 
Stande der Entwicklung in der Sowjetunion machte sich bei einzelnen Kollegen ein erhöhtes Verständnis für politökonomische Voraussetzungen bei der Entstehung gewisser exogener Noxen bemerkbar.

Die Veröffentlichung von Übersetzungen sowjetischer Wissenschaftler, die Fragen der höheren Nerventätigkeit, speziell solche der Neuropathologie und Psychiatrie, behandelten, wurde 1950 mit einem Beitrag über die Entwicklung der Ideen Pavlovs von Bykov sowie mit der Herausgabe des schon erwähnten Werkes von Speranskij [28] fortgesetzt. Seit 1951 folgten fachbezogene, ältere wie jüngere, immer zahlreicher, - einzelne, der deutschen Gesamtausgabe vorausgehende Ubbersetzungen Pavlovscher Schriften nicht ausgenommen. Von Ivanov-Smolenskij wurden nach mehr als einem Vierteljahrhundert erstmals wieder Arbeiten in Deutschland veröffentlicht. Mit zu den ersten Beiträgen zählte auch ein knapp auf die wichtigste fachliche Orientierung bemessener von S.N. Davidenkov (1881-1961). Seitens deutscher Kollegen setzte ebenfalls eine zunehmend regere Beteiligung an entsprechenden Erörterungen innerhalb und außerhalb des Fachgebiets ein [54].

Auf der ersten, dem Werk Pavlovs und seiner Schüler von Klinikern, Physiologen und Pharmakologen gewidmeten Tagung, die Anfang 1952 in Dresden stattfand, referierte als Vertreter der Psychiatrie Müller-Hegemann, der die Bedeutung der Resultate Bykovs, Ivanov-Smolenskijs und Krasnogorskijs charakterisierte und kritisch zu mißverständlichen Auslegungen der Pavlovschen Lehre und ablehnender Haltung ihr gegenüber Stellung nahm [55]. Fnde des Jahres erschien eine an Gesichtspunkten reiche Monographie über Psychotherapie bei schizophrenen Prozessen von ihm, in der er die Simonsche Arbeitstherapie würdigend erwähnte und außer auf Pavlov und Bykov eingehender auch auf Makarenko Bezug nahm [56]. Gleichzeitig kam die Übersetzung von Bykovs schon 1942 in der Sowjetunion veröffentlichtem Werk ,Großhirnrinde und innere Organe“" heraus [57], über das zuvor bereits referiert und diskutiert worden war.

Von einer international besuchten Tagung im Januar 1953, auf der $A . V$. Sne ̌nevskij (geb. 1904) die Wirkungen, die von Pavlov und seiner Schule auf die Psychiatrie ausgegangen waren, behandelte, nahmen die Fachkollegen starke Anregungen mit. Ein Referat, das Mïller-Hegemann, Pickenhain und E. Goetze (geb. 1913) ausgearbeitet hatten, widmete sich teilweise gleichfalls dieser Thematik, die von einem anderen Blickwinkel aus auch $H$. Kleinsorge, Jena (geb. 1920) streifte, der als Internist die Ätiologie und die Therapie funktioneller Krankheiten zum Gegenstand seiner Ausführungen gewählt hatte [58]. Der Ertrag der Aussprache für das Fachgebiet zeichnete sich in weiteren Fortschritten in der Überwindung biologistischer und nihilistischer Konventionen ab. Im Rahmen der unter Mithilfe der sowjetischen Besatzungsmacht eingeleiteten und inzwischen zum Teil vollzogenen Neugestaltung des Gesundheitsschutzes[59] erschien die Aneignung der Forschungsresultate der sowjetischen Neurophysiologie und Neuropathologie den um den Aufbau eines demokratischen humanen Gesundheitswesens bemühten Kräften als Komponente von ausschlaggebender Bedeutung. Dem entsprach es, daß von einer 
bein Ministerium für Gesundheitswesen gebildeten Staatlichen Pavlov-Kommission in dieser Hinsicht Unterstützung geboten wurde und daß ein in der Auswirkung sehr lebendiger Meinungstaustausch mit Fachvertretern in der Sowjetunion und in den volksdemokratischen Ländern sowie mit interessierten Kollegen aus kapitalistischen Staaten zustande kam [60].

Die Bereicherung, die das prophylaktische Denken, das Verständnis der Pathogenese und Pathodynamik der Symptomatik der Neurosen und Psychosen im einzelnen und die therapeutischen Methoden in den folgenden Jahren erfuhren, war groß und vielseitig. Ihren unmittelbaren Niederschlag fand sie literarisch in den Sitzungs- und Tagungsberichten der örtlichen und der zentralen Gesellschaften für Psychiatrie und Neurologie sowie für Psychotherapie und in zahlreichen Referaten und Beiträgen, die in der Mehrzahl laufend in dem Fachorgan „Psychiatrie, Neurologie und medizinische Psychologie" veröffentlicht worden sind [61]. In der Behandlung wurde der Arbeitstherapie und der Schlaftherapie ein bevorzugter Platz eingeräumt. Mit letzterer beschäftigte sich forschungsmäßig ganz allgemein das von Baumann geleitete, bereits erwähnte Institut. Praktisch fand sie über Universitätskliniken hinaus auch in städtische Krankenhäuser und in Landeskrankenhäuser Eingang. Ähnlich wie die Hypnose bildete sie häufig das Thema von Darlegungen und Diskussionen [62]. 1954 erschien Ivanov-Smolenskijs Buch .Grundzüge der Pathophysiologie der höheren Nerventätigkeit [63], zwei Jahre darauf Giljarovskijs Darstellung des Elektroschlafs [64] in deutscher Übersetzung.

Von drei Referaten, die Sne ̇nevski 1954 bei verschiedenen Anlässen in Leipzig, Berlin und Dresden hielt, bezog sich eins auf die Probleme der Schlaftherapie in der psychiatrischen Klinik [65], das nächste auf die psychogenen Psychosen und das letzte auf die Formen der senilen Demenz [66]. Zur Morphologie der Hirnrinde sprach in Leipzig zugleich S. A. Sarkisov (geb. 1885). Auf der Dresdner Tagung stand neben dem Referat Sneinevskis ein Beitrag, in dem Ozereckij über seine Arbeitsergebnisse berichtete. Die Bekanntschaft mit der sowjetischen Fachliteratur wurde inzwischen durch deutsche Ausgaben der Zeitschrift ,Sowjetisches Gesundheitswesen“ und der ,Pavlov-Zeitschrift für höhere Nerventätigkeit“", ferner auch durch das Erscheinen übersetzter Originalarbeiten in den Faltbeilagen des ,,Deutschen Gesundheitswesens" , für deren Redaktion sich namhafte Wissenschaftler zur Verfügung gestellt hatten. wesentlich gefördert. Von den Neuropathologen und Psychiatern der Sowjetunion traten außer den bereits genannten nun etliche weitere deutlicher ins Gesichtsfeld der Leser in der DDR; so z. B. K. I. Platonov (geb. 1877), P. $\boldsymbol{E}$. Snesarev (1876-1954), V. M. Bans̀ìikov (geb. 1898), I. V. Strel'iuk, V. K. Fedorov (1903-1963), D. D. Fedotov (geb. 1908). A. S. Cistovic (geb. 1897), durch sorgfältige Studien über Infektionspsychosen bekannt, befaßte sich mit der Störung der Wechselwirkung der Signalsysteme, die auch N.N.Traugott eingehend beschäftigte und $\mathrm{zu}$ einer aufschlußreichen monographischen Bearbeitung veranlaßte [67]. Zu der wichtigen Sparte der Psychiatrie der Kinder und der Jugendlichen vermittelten außer den Arbeiten Ozereckijs Schriften von G. E. Suchareva (geb. 1891), G. P. Simson (1892 -1960) und des schon in anderem Zusammenhang genannten viel- 
seitigen Forschers und Klinikers G.K. Ušakov, der sich längere Zeit in der DDR aufhielt und zu etlichen Fachkollegen persönlich in Beziehung trat, mit neuen Gesichtspunkten willkommene Verbindung.

Der Kontakt mit der Literatur der Nachbardisziplinen und einzelnen ihrer Experten war bei der engen Berührung, die zwischen dem Fachgebiet und der Physiologie der höheren Nerventätigkeit sowie angrenzenden Wissenschaftszweigen besteht, bald ziemlich rege. Zu den persönlich Anwesenden gehörten außer den schon als Tagungsgästen erwähnten damals K.M.Bykov, I. T.Kurcin (geb. 1907), L.G. Voronin, D. A. Birjukov (geb. 1904), Ch.S. Kośtojanc (1900-1961) sowie dann V. L. Balaksina (geb. 1910), G. V. Popov (geb. 1900), N. N. Sajko (geb. 1908) und N.P.Sinicyn (geb. 1900) als Gastprofessoren. Zum literarischen Werk, das parallel dazu mit im Vordergrund der Beachtung stand, zählten neben bereits direkt oder indirekt Angeführtem Schriften von P.S. Kupalov (1888-1964), L. A. Orbelo (1882-1958), P.K. Anochin (geb. 1898), F. A. Andreev (geb. 1879), A.T. Pšonik, E. Ś. Ajrapet'janc (geb. 1906), - um einige von vielen zu nennen. Auch Autoren aus anderen sozialistischen Ländern zollte man erhöhtes Interesse.

Das Streben nach einer konsequent monistischen Auslegung aller experimentellen und klinischen Beobachtungen veranlaßte einige Schüler und Anhänger Pavlovs aus den Reihen der Neuropathologen und Psychiater in der Zeit der heftigen Auseinandersetzungen, die sich 1950/51 unter Experten einzelner Wissenschaftszweige in der Sowjetunion abspielten, zur Kritik an Kollegen, die eine der dualistischen Betrachtungsweise entgegenkommende psycho-morphologische Interpretation befürworteten, und auch zur kritischen Stellungnahme gegenüber Schulen und Richtungen des Auslandes, die ähnlich verfuhren [68]. Der Meinungsaustausch mit Repräsentanten der Fachgesellschaft in der DDR blieb entsprechend der guten Aufnahme, die das sowjetische Gedankengut gefunden hatte, dadurch indessen unbehelligt. Von den scharfen Trennungsstrichen, die in der Polemik statuiert worden waren, wurde überdies hernach manches gemildert. Kliniker und Forscher wie Kraepelin und Kleist werden heute ohne Schmälerung ihrer Verdienste historisch gewürdigt. Auch Kollegen, die die Plastizität des Gehirns im Sinne Pavlovs ablehnen wie $K$. Leonhard, Berlin (geb. 1904), als Vertreter der Schule Kleists, gelten als wertvolle Bundesgenossen im Wirken für die Erneuerung der Psychiatrie [69]. Anders ist das Verhältnis gegenüber Autoren, die der auf medizinisch-naturwissenschaftlichen Grundlagen fußenden Psychiatrie den Rücken kehren und sich - wie es schon früh erkennbar wurde [44] - idealistischen Strömungen überantworten. Der sowjetische Kollege E. Sternberg (geb. 1903), bekannt durch seine gerontologisch-psychiatrischen Arbeiten, hat jüngst gezeigt, daß vor allem die ,,psychiatrische Anthropologie“ und die „Daseinsanalyse“ Fachvertretern in den Ländern mit monopolkapitalistischem Regime zu dem - freilich zum Scheitern verurteilten - Versuch dienen, spekulative Konzeptionen des Idealismus in die Medizin und insbesondere in die Psychiatrie hereinzutragen [70].

Als ab 1953 die ermutigenden Erfolge der medikamentösen Verabreichung von von Phenothiazinderivaten im einschlägigen Schrifttum international erörtert 
wurden und beachtliche Resultate bei Gebrauch weiterer neuer Pharmaka die Fachexperten beglückten, verringerte sich das Interesse an den übermittelten Methoden und der Theorie der sowjetischen Medizin nicht. Wenn ihre Aktualität Einbuße erlitt, so höchstens infolge des Umstandes, daß ihren vorbildlichen schonungsvollen Behandlungskombinationen, über die das 1960 in deutscher Übersetzung erschienene Lehrbuch von Giljarovskij [71] erneut umfassend unterrichtete, nun andere an die Seite traten, die den Vergleich eher aushielten als die früheren. Der Berücksichtigung der Gesetzmäßigkeiten der höheren Nerventätigkeit in der Neurophysiologie und Neuropathologie war allgemein eine Vertiefung und Verfeinerung der klinischen Kenntnisse der Neurosen und der Psychosen [72] zu verdanken, die sowohl zur Überwindung der Freudschen Doktrin vom Unbewußten als zu der der phänomenologischen Denkweise führten.

Die sowjetische Psychiatrie ist als Zweig der Medizin des ersten sozialistischen Staates dank engerer Beziehung zur Pavlovschen Lehre für die in unserem Arbeiter-und-Bauern-Staat neugestaltete Fachrichtung zum Vorbild, zur Quelle mannigfaltiger Bereicherung und zum Hebel für die Abkehr von den Methoden und Anschauungen geworden, die Einflüsse der Ideologie des Imperialismus erkennen ließen. Der Vielfalt der Auswirkungen und den Leistungen der Personen, auf die sie sich erstreckten, gerecht zu werden, könnte nur einer umfangreicheren Darlegung gelingen. Die Anregungen zu einer dem dialektischen Materialismus gemäßen Klärung der wissenschaftlichen und organisatorischen Probleme im Fachbereich haben dazu verholfen, daß die Wurzeln der früher in der Symptomatologie, der Nosologie, der Typologie und in der Psychotherapie vorherrschenden Konjekturen bloßgelegt wurden. Außerdem bildete gerade in diesem Wissenschaftszweig die nähere Bekanntschaft mit der Pavlovschen Lehre Ansätze zum Verständnis der weiteren neuen Feststellungen heraus, die die Neurokybernetik zu erschließen verheißt [73].

\section{Literatur}

1. I. Winter, Georg Benjamin, Arzt und Kommunist, Berlin 1962.

2. I. Winter: Zur Geschichte der Gesundheitspolitik der deutschen Arbeiterbewegung. NTM dieses Heft S. 93.

3. Ein Besuch im Psychiatrischen Krankenhaus ,,Kastschenko“" in Moskau. Der Sanitäter. Jg. 1, H. 3. Berlin 1926.

4. A. Mette: Wilhelm Griesinger, in: Von Liebig bis Laue, hrsg. von O. Finger und Fr. Herneck. Berlin 1963.

5. Kl. Fichtel: Wilhelm Griesinger - ein Vorläufer der materialistischen Reflextheorie. Z. ärztl. Fortb. Jg. 59, H. 18. 1965.

6. L. Pickenhain: Grundriß der Physiologie der höheren Nerventätigkeit. Berlin 1959.

7. I. P. Pavlov: Sämtliche Werke. Dtsch. Ausg. Gesamt-Redkt. L. Pickenhain. Berlin 1953/54.

8. Ivan Petrovitsch Pavlov (chronologisches Verzeichnis seiner Arbeiten). Dtsch. Ausg. Redkt. K. Winter.

9. Pavlov: Werke Bd. VI, S. 229.

10. J. M. Ufljand: Die Hauptetappen in der Entwicklung der Lehre N. Je. Wedenskis. Berlin 1955.

11. A. A. Uchtomski: Erregung, Ermüdung, Hemmung. Aus d. Schriftt. d. Sowjetunion und d. Volksdemokratien. Dtsch. Ges.wesen, Jg. 10, Faltblg. s. 177 ff. 1955. 
12a. A. Czerny: Über Bedingungsreflexe im Kindesalter. Straßburger med. Zt. Jg. 7, S. $230 \mathrm{ff} .1910$.

12b. A. Peiper: Die Hirntätigkeit des Säuglings. Berlin 1928.

13. K. Kleist: Die gegenwärtigen Strömungen in der Psychiatrie. Berlin u. Leipzig 1925.

14. O. Bumke: Über die gegenwärtigen Strömungen in der klinischen Psychiatrie, in: Die gegenwärtigen Strömungen in der Psychiatrie. Berlin 1928.

15. K. Schneider: Psychiatrie heute. Stuttgart 1952.

16. Bericht über den 3. Physiologenkongreß in Bern. Zbl. Physiol., Jg. 9, S. 465, 1895.

17. A. Mette: Porträt eines Humanisten. ,Aufbau', kulturpolitische Monatsschrift, Jg. 10, H. 9, 1954.

18. A. Mette: Sigmund Freud, Berlin 1956.

19. O. Bumke: Handbuch der Geisteskrankheiten. Berlin 1932.

20. A. R. Luria: Die moderne russische Physiologie und die Psychoanalyse. Int. Ztschr. Psychoanalyse, Jg. XII, H. 1. Wien 1926.

21 a. W. Reich: Dialektischer Materialismus und Psychoanalyse. Unt. d. Bann. Marxim. III. H. 5, 1929.

21 b. A. Mette: Zur marxistischen Kritik der Psychoanalyse. ,Aufbau', Erziehungswissensehaftliche Zeitschrift, Jg. 3, H. 3, 1930.

22. I. Sapir: Freudismus Soziologie Psychologie. Unt. d. Bann. d. Marxism. Jg. III, H. 6, Jg. IV, H. 1. Wien 1929/30.

23. J. Streisand: Max Weber: Politik, Soziologie und Geschichtsschreibung, in: Studie über die deutsche Geschichtswissenschaft, hrsg. von J. Streisand, Bd. II. Berlin 1965.

24. H. Berger: țber das Elektro-Encephalogramm des Menschen. Arch. f. Psychiat., Bd. 87, 1929.

25. L. Pickenhain: Grundriß S. 90-101.

26. E. R. Jaensch: Der Gegentypus. Leipzig 1938.

27. A. Mette: Die tiefenpsychologischen Grundlagen des Tragischen, Apollinischen und Dionysischen. Berlin 1934.

28. A. D. Speransky: Grundlagen der 'Theorie der Medizin. Dtsch. Ausg. Rdkt. K. von Roques. Berlin 1950.

29. Pavlov: Werke Bd. III.

30. I. P. Pavlov: Mittwochskolloquien. Dtsch. Ausg. Rdkt. L. Pickenhain S. $6 \mathrm{ff}$. Berlin $195 \check{.}$

31. Izvestija v. 4. 2. 1936, Pravda v. 4. 2. 1936.

32. A. G. Ivanov-Smolenski: Untersuchungen über das wechselseitige Zusammenwirken des ersten und des zweiten Signalsystems, angewandt auf die Aufgaben der Medizin. Sowj. wissensch., Naturwiss. Abt. 2 (1954) S. $173 \mathrm{ff}$.

33. А.Г.Иванов-Смоленский: Оснорные Іроб̄лемы Патофизиологии высшей первной деятельности (Grundprobleme der Pathophysiologie der höheren Nerventätigkeit). Biomedgis 1933.

34. M. Cloetta und H.W. Maier: Über eine Verbesserung der psychiatrischen Dauernarkosebehandlung. Ztschr. f. d. ges. Neurol. u. Psych. Bd. 150, H. 1, 1934.

35. E. H. Ackerknecht: Kurze Geschichte der Psychiatrie. Stuttgart 1957.

36. 3. А. Асратян: Сониая терапия травматических Поражкений Нервной системы (Die Schlaftherapie traumatischer Verletzungen des Nervensystems). Sonderdruck 1943.

37. 3. А. Асратян: К Теории и практике охранительной и целебной ролиторможения

(Zur Theorie und Praxis der schützenden und heilenden Bedeutung derHemmung). Physiol. Journ. d. UdSSR. Bd. XXXII, H. 1, 1946.

38a. Schmerzausschaltung bei der Geburt. Abhandlg. einer Konferenz i. Leningrad 1951, eingel. v. N. N. Anickov. Dtsch. Ausg. Rdkt. Ruppert, Fichtel, Pickenhain, Berlin 1953.

38 b. F. A. Andrejev: Krankheitsbeginn. Dtsch. Ausg. Rdkt. H. Eckstein. Berlin 1955.

39. W. H. Veil und A. Sturm: Die Pathologie des Stammhirns. 2. Aufl. Jena 1946.

40. W. H. Veil: Goethe als Patient. 2. Aufl. Jena 1946.

41. H. Scliwarz: Ein Gutachten über die ärztliche Tätigkeit im sog. Erbgesundheitsverfahren. Samml. zwangl. Abhandlg. aus d. Ciebiet d. Psychiatrie u. Neurologie, hrsg. von H. Schwarz. Halle 1950.

42. E. A. Asratjan, Anpassungsvorgänge im geschädigten ()rganismus. Dtsch. Ges.wesen Jg. 2, H. 15. 
43. Wissensch. Tagung d. Psychiater u. Neurologen i. d. S. B. Z. D. Tagungsbericht. Psychiat. Neurol. med. Psychol. Jg. 1, H. 2, 1949.

44. A. Mette: Der ,Blick in die eigene Tiefe", Forschungen u. Fortschritte Jg. 25, H. 7/8, 1949.

45. A. Mette: Bemerkungen zu Freuds Begriff der Regression. S. S. Korsakow-Journ. f. Neuropath. u. Psychiat. Jg. 58, H. 1, 1958.

46. Psychiatrie, Neurologie und medizinische Psychologie. Zeitschrift für Forschung und Praxis, hrsg. von A. Mette, Jg. 1, 1949.

47. R. Baumann: Physiologie des Schlafes und Klinik der Schlaftherapie. Berlin 1953.

48. G. Göllnitz: Ergebnis einer ¿̇̉berprüfung der motometrischen Skala von Oserezki. Psychiat. Neurol. med. Psychol. 4. Jg., H. 5, $19 \tilde{2}$.

49a. Betr.: I. P. Pavlov, sämtliche Werke.

49 b. Ein Dokumentationszentrum widmete sich der Veröffentlichung der medizinisch-wissenschaftlichen Literatur als Beratergruppe.

50. A. Mette: Bemerkungen über den gegenwärtigen Zustand der Psychiatrie in der DDR, S. S. Korsakow-Journ. f. Neuropath. u. Psychiat. Jg. 57, H. 1, 1957.

5l. (9. K. Ušakov: О Путях развития и направлениях исследований в психонервологических клиниках Германской Демократической Респуо̆тики

(Über die Wege der Entwicklung und die Richtungen der Forschung in den psychiatrischen Kliniken der DDR). Moskau 1964.

52a.D.Müller-Hegemann:ZweiWege in der modernenPsychotherapie.Dtsch.Ges.wesen.Jg.6,H.23,1951. 52b. D. Müller-Hegemann: Über die Bedeutung der neurologisch-psychiatrischen Fachrichtung in der Gegenwartsmedizin, in: Probleme der Neurologie und der Neuropathologie im Lichte der Lehre I. P. Pavlovs. Berlin 1952.

52c. D. Müller-Hegemann: Neue Wege der psychiatrisch-neurologischen Forschung auf Grund der Arbeiten der Pavlovschen Schule. Psychiat. Neurol. med. Psychol. Jg. 7, H. 7, 1955.

52d. L. Pickenhain: Um die Anwendung der Lehre Pavlovs in der deutschen Medizin. ,Aufbau', Kulturpolitische Monatsschrift Jg. 10, H. 4, 1954.

52e. A. Mette: Spekulation und exakte Wissenschaft in der Typenlehre. Erste Arbeitstagung über zentrale Regulation der Funktionen des Organismus (vgl. 60 b). Berlin 1956.

53a. W. Hollitscher: Die Lehre Pavlovs - die Grundlage der wissenschaftlichen Physiologie und Psychologie. Einheit Jg. 7, H. 6, 1952.

53b. L. Pickenhain: Die Lehre Pavlovs - ein Beweis für den dialektischen Materialismus. Wiss. Ztschr. d. Humboldt-Universität z. Berlin. Math. nat. Reihe Jg. 3, Nr. 4, $1953 / 54$.

53c. A. Thom: Philosophisches Denken in der modernen Psychiatrie. Wiss. Ztschr. d. Karl-MarxUniversität zu Leipzig. Math. nat. Reihe, Sonderband V, Medizin und Philosophie, Arzt und Gesellschaft 1965.

54. Die Lehre I. P. Pavlovs, Bibliographie deutschsprachiger Veröffentlichungen zus.gest. v. G. Krause u. W. Dube (mit laufenden Nachträgen als Beilagen zumDtsch. Ges.wesen). Berlin 1955.

55. Über das Werk I. P. Pavlovs und seiner Schüler, Tagungsber. d. Tagung in Dresden am 9. Febr. 1952, Leitung von E. von Skramlik. Berlin 1952.

56. D. Müller-Hegemann: Die Psychotherapie bei schizophrenen Prozessen. Leipzig 1952.

57. K. M. Bykov: Großhirn und innere Organe. Dtsch. Ausg. hrsg. v. M. Zetkin. Berlin 1952.

58. Pavlov-Tagung Leipzig 15. u. 16. Jan. 1953. WViss. Ltg. D. Müller-Hegemann, A. Peiper, S. Rapoport, M. Zetkin. Berlin 1953.

59. Die Bedeutung der Befehle der SMAD für den Aufbau des sozialistischen Gesundheitswesens der Deutschen Demokratischen Republik. Berlin 1967.

60a. Arbeitstagung über kortikoviszerale Regulationen Leipzig 15. bis 17. Jan. 1954. Wiss. Ltg. F'r. Jung, H. Kleinsorge, D. Müller-Hegemann, Berlin 1955.

60 b. Erste Arbeitstagung über zentrale Regulation der Funktionen des Organismus. Leipzig 1. bis 3. Dez. 1955 Wiss. Ltg. P. Hertwig, A. Mette, S. Rapoport Berlin 1956.

61. 1959 traten K. Leonhard und D. Müller-Hegemann mit in die Herausgeberschaft der 1949 gegründeten Zeitschrift (vgl. 46) ein. 
62a. K. I. Platonov: Suggestion und Hypnose im Lichte der Lehre I. P. Pavlovs. Dtsch. Ausg. Rdkt. K. Winter, Berlin 1953.

62 b. H. Kleinsorge u. G. Klumbies: Technik der Hypnose für Ärzte. Jena 1961.

62 c. H. Kleinsorge u. G. Klumbies: Technik der Relaxation. Selbstentspannung. Jena 1961.

62d. F. Völgyesi: Az Orvosi Hipnózis. Budapest 1962.

62e. F. Völgyesi: Hypnose bei Mensch und Tier. Dtsch. Ausg. Redkt. G. Klumbies. Leipzig 1967.

62f. R. Baumann: Die Schlaftherapie. Referat auf d. Arbeitstagung über kortikoviszerale Regulationen (vgl. 60a).

62 g. D. Müller-Hegemann: Erfahrungen mit der Schlaftherapie. Med. Klin. Jg. 49, H. 10, 1954.

62h. A. Mette: Schlaftherapie und Schutzregime. Heilkunst Jg. 67, H. 10, 1955.

63. A. G. Ivanov-Smolenski: Grundzüge der Pathophysiologie der höheren Nerventätigkeit. Dtsch. Ausg. Rdkt. U. Bauer. Berlin 1954.

64. V. A. Giljarovski, N. M. Livencev, J.J. Segal und S. A. Kirillova: Elektroschlaf. Dtsch. Ausg. Red. Ltg. H. Kleinsorge.

65. A. W. Snežnevski: Probleme der Schlaftherapie in der psychiatrischen Klinik. Referat auf d. Arbeitstagung über kortiko-viszerale Regulationen (vgl. 60a).

66a. A. W. Snežnevski: Die psychogenen Psychosen. Referat in d. Berliner Charité Jan. 1954. Dtsch. Übers. Rdkt. K. Fichtel. Dtsch. Ges.wesen Jg. IX Faltbeil. S. 169.

66 b. A. W. Snežnevski: Die Formen der senilen Demenz. Referat auf d. Psychiater-NeurologenTagung der DDR. Dresden 12. Okt. 1954. Dtsch. Ausg. Rdkt. L. Pickenhain. Dtsch. Ges.wesen Jg. IX, Failtbeil. S. 569 .

67. N. N. Traugott: Störungen der Wechselwirkungen der Signalsysteme. Dtsch. Ausg. Übers./Rdkt. H. Lammert.

68. Die physiologische Lehre des Akademiemitgliedes I. P. Pavlov in Psychiatrie und Neuropathologie. Tagungsbericht. Dtsch. Ausg. Rdkt. P. G. Klemm. Berlin 1956.

69. Актуальные пробллемы психиатрии (Aktuelle Probleme der Psychiatrie), Red. Ltg. D. D. Fedotov. Moskau 1959.

70. $E$. Sternberg: Zur Kritik der existentialistischen und anderen antinosologischen Richtungen in der modernen westlichen Psychiatrie. Psychiat. Neurol. med. Psychol. Jg. 16, H. 11, 1964.

71. V. A. Giljarovski: Lehrbuch der Psychiatrie. Dtsch. Ausg. Übers./Rdkt. H. Lammert, Berlin 1960. 72a. D. Müller-Hegemann: Psychotherapie. Berlin 1957.

72 b. Neurosenprobleme in Klinik und Experiment. Ausgew. Vortr., Fortb. Lehrg. 25. bis 28. Juni 1958, Ltg. D. Müller-Hegemann, Schriftenr. d. ärttl. Fortb. Hrsg. von H. Redetzky u. H.Thiele, Berlin 1959.

72c. E. Schmidt-Kolmer: Erscheinungsformen des psychischen Hospitalismus in den ersten Lebensjahren und ihre Bekämpfung. Psychiat. Neurol. med. Psychol., Jg. 11, H. 8, 1959.

72d. A. Mette: Über Angstzustände anläßlich der Unsicherheit über die Identität von Personen beim Kleinkind. Ebenda Jg. 6, H. 12, 1954.

72e. A. Mette: Bemerkungen zur Symptomatik und Theorie der Sprachverwirrtheit bei Schizophrenie. Referat i. d. Ges. f. Neurol. u. Psychiat. a. d. Univ. Berlin 13. 12. 1954. Ebenda Jg. 7, H. 3, 1955.

72f. A. Mette: Die Sprache der Schizophrenen. Referat auf d. Psychiater-Neurologen-Tagung der DDR. Dresden 1. bis 3. Nov. 1956. Dtsch. Ges.wesen, Jg. 12, H. 1, 1957.

72g. K. Zaimov: Über die Pathophysiologie der Agnosien, Aphasien, Apraxien und der Zerfahrenheit des Denkens bei der Schizophrenie. Sammlg. zwangl. Abhandl. a. d. Gebiete d. Psychiat. u. Neurol., hrsg. v. H. Schwarz, Jena 1965.

72h. D. Müller-Hegemann: Über bedingt-reflektorische Therapie. Referat a. d. 3. Weltkongreß d. Psychiatrie, Montreal 7. Jun. 1961. Psychiat. Neurol. med. Psychol. Jg. 14, H. 1, 1962.

72i. D. Müller-Hegemann: Neurologie und Psychiatrie. Berlin 1966.

73a. Arzt und Philosophie. Symposium anl. d. 150jähr. Jubiläums der Humboldt-Universität u. d. 250jähr. Jubiläums der Charité, Berlin, 7. bis 9. Nov. 1960. Ltg. F. Jung, G. Klaus, A. Mette, S. M. Rapoport. Berlin 1961.

73 b. S. N. Braines, A. V. Napalkov, V. B. Swečinski: Neurokybernetik. Mit einer Einf. von G. Klaus. Berlin 1964. 